\title{
In Vitro Biomechanical Evaluation of a Novel, Minimally Invasive, Sacroiliac Joint Fixation Device
}

\author{
WILLIAM W. CROSS III, MD, ${ }^{1}$ SIGURD H. BERVEN, MD ${ }^{2}$ NICK SLATER, MS, ${ }^{3}$ JENNIFER N. LEHRMAN, \\ MS, ${ }^{4}$ ANNA G. U. S. NEWCOMB, MS, ${ }^{4}$ BRIAN P. KELLY, PHD ${ }^{4}$ \\ ${ }^{1}$ Department of Orthopedic Surgery, Mayo Clinic, Rochester, Minnesota, ${ }^{2}$ Department of Orthopedic Surgery, University of California at San Francisco, San \\ Francisco, California, ${ }^{3}$ CoorsTek Medica, Chandler, Arizona, ${ }^{4}$ Spinal Biomechanics Laboratory, Barrow Neurological Institute, St. Joseph's Hospital and Medical \\ Center, Phoenix, Arizona
}

\begin{abstract}
Background: Sacroiliac (SI) joint pathology may result in low-back pain, which causes substantial disability. Treatment failure with operative management of SI pain may be related to incomplete fusion of the joint and to fixation failure. The objective of this study was to evaluate the initial biomechanical stability of SI joint fixation with a novel implantable device in an in vitro human cadaveric model.

Methods: The right and left sides of 3 cadaveric L4-pelvis specimens were tested (1) intact, (2) destabilized, and (3) instrumented with an implantable SI joint fixation device using a simulated single-stance load condition. Right-leg and left-leg stance data were grouped together for a sample size of 6, and angular range of motion (ROM) was determined during application of flexion-extension, lateral bending, and axial rotation bending moments to a limit of $7.5 \mathrm{Nm}$.

Results: Following intact testing, destabilization by severing the posterior SI joint capsule and ligaments and the pubic symphysis reliably produced a significantly destabilized joint with the mean angular ROM more than doubling in flexion-extension and lateral bending and more than tripling in axial rotation $(P \leq .003)$ compared to the intact condition. Instrumentation with the SI screw fixation device significantly reduced mean joint ROM compared to the destabilized condition in all 3 anatomic planes tested $(P<.001)$. When compared to the intact condition, the SIinstrumented condition significantly reduced lateral bending $(P=.01)$ and had a similar ROM in flexion-extension $(P=$ $.14)$ and axial rotation $(P=.85)$.

Conclusions: Instrumentation with the SI screw fixation device significantly reduced mean joint ROM compared to the destabilized condition, with similar ROM in flexion-extension and axial rotation, and it significantly reduced ROM in lateral bending compared to that for the intact joint. The ROM values observed with the instrumented condition were comparable to levels of mobility considered favorable for spinal fusion.
\end{abstract}

Biomechanics

Keywords: biomechanics, fusion, lag screw, range of motion, sacroiliac joint, single-leg stance, stability

\section{INTRODUCTION}

Pain in the lower back has multifactorial causes, and a broad spectrum of disorders may lead to the common clinical presentation of low-back pain. Pain of spinal origin may be related to pathology of the intervertebral disc, facet joints, or sacroiliac (SI) joint. ${ }^{1}$ The SI joint is an important source of axial back pain, which causes substantial disability., The burden of SI pain may be measured by the number of patients affected by SI disease and by the impact of SI disorders on health-related quality of life. Both prevalence and impact of disease estimates confirm that pathology of the SI joint presents a significant burden to our health care economy. ${ }^{4}$

Management of pain from the SI joint is variable and encompasses nonoperative and operative ap- proaches. ${ }^{5}$ Minimally invasive surgical approaches to the SI joint have increased dramatically in the past 5 years, with increased recognition of SI disorders as a source of pain and the development of systems for surgical stabilization and fusion of the SI joint. ${ }^{6}$ However, efficacy of operative management of SI pain has been variable. ${ }^{7-9}$ Failure rates as high as $50 \%$ in some series may be related to incomplete fusion of the SI joint and failure of fixation. ${ }^{10}$

The SI joint is a large, diarthrodial joint with dense innervation and a complex motion pattern. ${ }^{11-13}$ Arthrodesis of the SI joint is challenging, and high rates of failure have been reported with open and minimally invasive approaches. ${ }^{14-16}$ The principles of arthrodesis require decortication of adjacent bone 


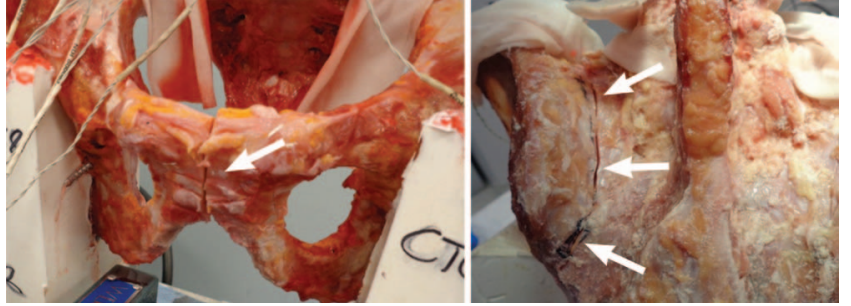

Figure 1. (Left) Anterior view of a destabilized specimen with cut pubic symphysis (arrow). (Right) Posterior view of a destabilized specimen with cut posterior sacroiliac joint capsule and ligaments (arrows). Used with permission from Barrow Neurological Institute, Phoenix, Arizona.

surfaces, compression across the joint, and rigid internal fixation. ${ }^{17}$ A novel screw fixation system for arthrodesis of the SI joint, Integrity-SI (CoorsTek Medical, LLC, Fort Worth, Texas), has been designed to optimize these principles of joint fusion. However, the compressive and stabilizing potential of this design has not been investigated. The objective of this study was to evaluate the biomechanical stability of initial SI joint fixation with this novel device in an in vitro human cadaveric model.

\section{MATERIALS AND METHODS}

\section{Specimen Preparation}

Three human cadaveric specimens ( 2 female, 1 male) with a mean \pm SD age of $46.7 \pm 7.5$ years were studied. Specimens were transected rostrally at L4 and included the lower lumbar spine, sacrum, and pelvis. Computed tomography (CT) scans of specimens were reviewed by an orthopedic surgeon (W.W.C.) to ensure that no specimen had obvious radiographic abnormalities. Similarly, the medical histories of the 3 individuals were reviewed to exclude any with metastatic disease, osteophytes, disc narrowing, or joint arthrosis. Dual energy x-ray absorptiometry scans performed on the L4 vertebra of each specimen yielded a mean bone mineral density value of $0.742 \pm 0.134 \mathrm{~g} / \mathrm{cm}^{2}$.

Specimens were obtained fresh frozen, then thawed at room temperature and carefully cleaned of muscle tissue without damage to any ligaments, discs, or joint capsules, including the pubic symphysis. Specimens were wrapped in plastic bags and stored in a freezer at $-20^{\circ} \mathrm{C}$ until tested. In preparation for testing, specimens were thawed in room temperature physiologic saline. Wood screws were inserted in various locations in the exposed L4 end plate and facet articulations. These screw heads were embedded in fast-curing resin (Smooth-Cast 300Q, Smooth-On, Inc, Easton, Pennsylvania) in a

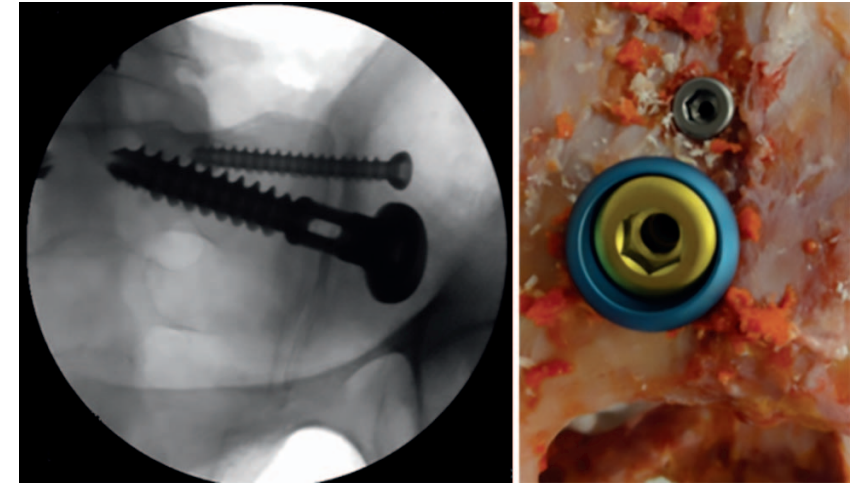

Figure 2. (Left) Radiographic image and (right) photograph of cadaveric specimen implanted with a sacroiliac joint implant comprising a lag screw, an antirotation screw, and a large adjustable washer. Used with permission from Barrow Neurological Institute, Phoenix, Arizona.

cylindrical potting fixture for application of loads. Similarly, screws were inserted near the right and left ischia on the pelvis, and the screw heads were embedded in 2 separate (right and left) blocks of resin. Each resin block was rectangular in shape such that it could be clamped in a vise and securely attached to the base of a servohydraulic test frame (MTS Systems Corp, Minneapolis, Minnesota) while still allowing free access to instrumented sites.

\section{Testing Conditions}

The right and left sides of each specimen were tested in the following 3 conditions: (1) intact, (2) destabilized, and (3) instrumented with an implantable SI joint fixation device. The destabilization procedure was performed by a neurosurgical resident and consisted of complete sectioning of the pubic symphysis in the sagittal plane and sectioning of the posterior SI joint capsule and ligaments (Figure 1). For the instrumented condition, the Integrity-SI joint fixation system was used (Figure 2). Briefly, this system comprises a large "lag"-type screw with a hollowed shank for graft placement, a variable-angle washer system with a large footprint, and a smaller antirotation screw. The implants were selected and placed by an orthopedic surgeon (W.W.C.) under fluoroscopic guidance, following the manufacturer's recommended surgical technique. For the antirotation screw, a sizing of 6.5$\mathrm{mm}$ diameter by $40-\mathrm{mm}$ length was chosen for 5 of the 6 SI joint specimens, and the sixth specimen received a 50-mm-long screw. For the larger screw, 5 of the 6 SI joints received a 12-mm-diameter by 70mm-long screw, and the sixth specimen received a slightly longer screw (80 $\mathrm{mm}$ long). 


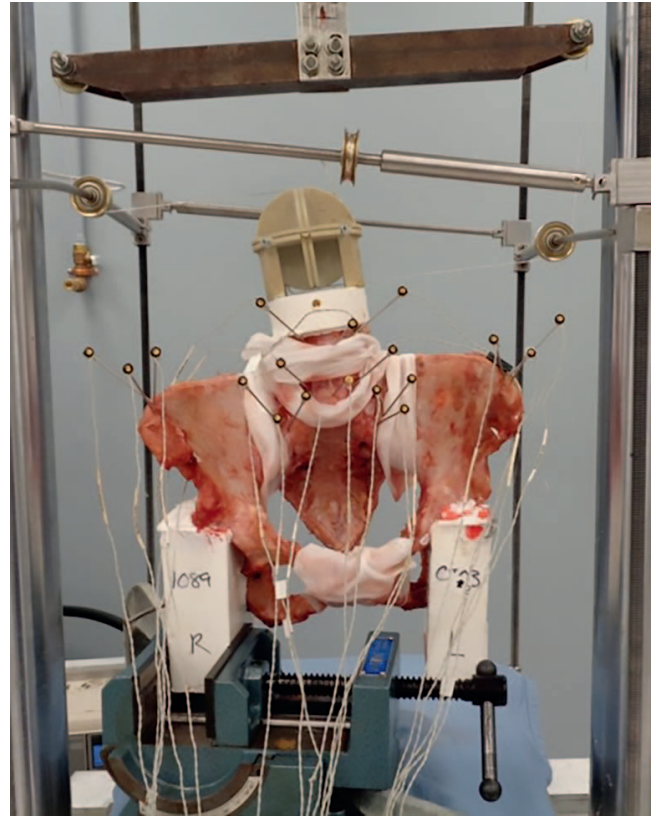

Figure 3. Biomechanical flexibility testing setup. Anterior view of a potted specimen in the test frame under load. For simulation of single-stance loading, the resin block attached to the acetabulum on the right side was clamped in a vise rigidly attached to the bottom of the test frame. The resin block attached to the acetabulum on the left side was left to hang freely. Desired directional loads within anatomic planes were induced by reorientation of the cranially attached cable and pulleys. Used with permission from Barrow Neurological Institute, Phoenix, Arizona.

The surgical technique involved preoperatively templating the optimal trajectory for the implant into either the upper or the lower sacral segment, pending elements of sacral dysmorphism. ${ }^{18} \mathrm{~A}$ cannulated system was used to obtain the appropriate starting point for undertaking access to the SI joint. Appropriate fusion device length was measured and placed across the SI joint. Once the main implant was placed, a cannulated device was again used to place the antirotation screw based on the trajectory of the first implant. The torque applied to seat the implants was at the discretion of the surgeon. No torque gauge or torque limiter was used in seating the implants. The lateral wall of the ilium was not violated by the washer when the final implant seating was achieved.

\section{Biomechanical Testing}

Left and right sides of each specimen were tested sequentially in each of the 3 conditions using a simulated single-leg stance load condition. This condition was modeled by rigidly clamping the resin block attached to the ipsilateral acetabulum in the vise and leaving the resin block attached to the acetabulum on the contralateral side unconstrained
(Figure 3). For all tests, an apparatus was used in which a system of cables and pulleys imparted nondestructive, nonconstraining torque in conjunction with a standard servohydraulic test system (MTS Systems Corp), as described previously by Crawford et $\mathrm{al}^{19}$ ). Pure moment loads were applied and distributed evenly to each motion segment regardless of the distance from the point of loading. ${ }^{20}$ Loads of $7.5-\mathrm{Nm}$ maximum were applied about the appropriate anatomic axes to induce 3 different types of motion: flexion-extension, lateral bending, and axial rotation. Three preconditioning cycles were applied at $7.5 \mathrm{Nm}$ for 60 seconds to allow for creep in each loading direction to ensure appropriate settling at the instrument-bone interface and to improve the reproducibility of the results. Specimens were kept moist during testing by using saline-soaked gauze wrapped around the SI joints and intervertebral discs.

Three-dimensional specimen motion in response to the applied loads during flexibility tests was determined using the Optotrak 3020 system (Northern Digital, Inc, Waterloo, Ontario, Canada). This system stereophotogrammetrically measured the 3dimensional displacement of infrared-emitting markers rigidly attached in a noncollinear arrangement to 6 regions on the lumbar spine and pelvic ring, namely, L4, L5, sacrum (2 locations, 1 right and 1 left), and the right ilea and the left ilea. A coordinate system for each set of marker triadsand hence for each motion segment - was defined through a manual digitization process that aligned each system with respect to the specimen's L5 anatomy. Custom software converted the raw marker coordinates to angles about each of the anatomic axes in terms of the motion segment's own coordinate system. ${ }^{21}$ Angles (ie, rotation of the right sacrum relative to the right ilium and of the left sacrum relative to the left ilium during flexionextension, right lateral bending and left lateral bending, and right axial rotation and left axial rotation) were calculated using a technique that provides appropriate results for describing 3-dimensional motion. ${ }^{22}$

\section{STATISTICAL METHODS}

From the raw data, we determined angular range of motion (ROM) from recorded quasi-static loaddeformation data. Larger values of ROM indicate greater joint instability. For simplicity and to avoid confusion in comparing right versus left single-leg 
Table 1. Angular range of motion at the sacroiliac (SI) joint for all conditions during single-leg stance. ${ }^{a}$

\begin{tabular}{lccc}
\hline Loading Direction & Intact $(\mathbf{n}=\mathbf{6})$ & ${\text { Destabilized }(\mathbf{n}=\mathbf{6})^{\mathbf{b}}}^{\text {S }}$ & SI-Instrumented Fixation $(\mathbf{n}=\mathbf{6})$ \\
\hline Flexion-extension & $2.92 \pm 0.74$ & $6.16 \pm 2.11$ & $1.75 \pm 0.99$ \\
Lateral bending & $1.16 \pm 0.39$ & $2.62 \pm 0.44$ & $0.59 \pm 0.34$ \\
Axial rotation & $1.46 \pm 0.27$ & $5.36 \pm 1.09$ & $1.52 \pm 0.67$ \\
\hline
\end{tabular}

${ }^{\mathrm{a}}$ Values are mean $\pm \mathrm{SD}$.

${ }^{\mathrm{b}} \mathrm{Cut}$ posterior ligament capsule and pubic symphysis.

stance, we studied only bidirectional values of ROM (eg, ROM during lateral bending for a single SI joint was the combination of applied bending to the right and left [ie, abduction + adduction]). Furthermore, analysis of bidirectional ROM allowed leftleg and right-leg stance data to be grouped together for a total sample size of 6 (3 right and 3 left sides).

Mean values of ROM were statistically analyzed using 1-way repeated-measures analysis of variance (RM-ANOVA) to determine whether there was a significant difference in mean parameter values for the different conditions. This analysis was followed by pairwise Holm-Sídák tests to determine which pairs of conditions differed. $P$ values less than .05 were considered statistically significant.

\section{RESULTS}

The mean \pm SD ROM for the intact, destabilized, and instrumented conditions are summarized in Table 1 and presented graphically in Figure 4. $P$ values from the 1-way RM-ANOVA and from the

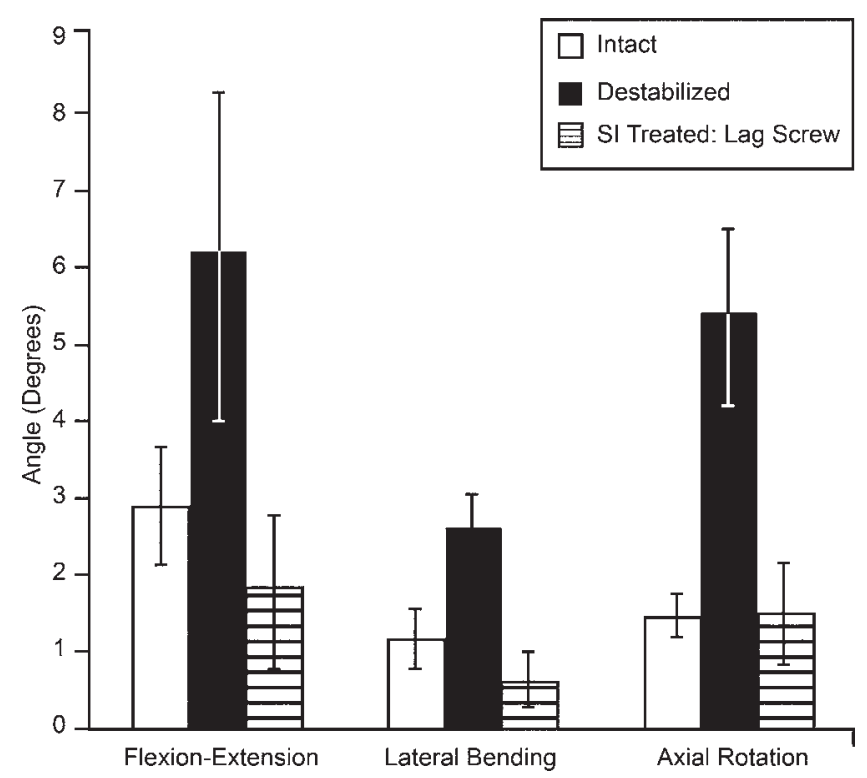

Figure 4. Mean angular range of motion observed at the sacroiliac (SI) joint during all test conditions. Error bars indicate standard deviations. Used with permission from Barrow Neurological Institute, Phoenix, Arizona.
Holm-Š́dák tests, comparing the various conditions, are summarized in Table 2. The mean intact angular ROM more than doubled in flexionextension and lateral bending, and it more than tripled in axial rotation to values of $6.2^{\circ}, 2.6^{\circ}$, and $5.4^{\circ}$, respectively, following destabilization of the specimens. The increased mobility was statistically significantly different during all directions of motion compared to the mobility of intact joints $(P \leq .003)$. Compared to the destabilized state, the SI-instrumented condition decreased mobility significantly ( $P$ $<.001)$, with a decrease of more than two-thirds in angular ROM in all directions of testing. Mean stability in the SI-instrumented condition was less than that for the baseline intact condition but was not statistically significant during flexion-extension $(P=.14)$. SI-instrumented stability was significantly lower than the intact condition during lateral bending $(P=.01)$. Stability with the SI-instrumented condition was quite similar to that of the intact condition during axial rotation $(P=.85)$.

\section{DISCUSSION}

A single-leg stance in vitro testing protocol ${ }^{23,24}$ was used to characterize the SI joint fixation stability of a novel implantable screw fixation device. This protocol was previously shown to produce intact SI joint angular ROMs that are in very good agreement with published reports of physiologic in vivo SI joint motion, with the greatest values occurring in flexion-extension, followed by axial rotation, and the least ROM observed in lateral bending. ${ }^{23}$

After intact testing, severing of the posterior SI joint capsule and ligaments as well as the pubic symphysis was effective in reliably producing a destabilized joint condition with significant increases in ROM in all 3 anatomic planes of testing while still retaining sufficient mechanical integrity to consistently withstand preconditioning and data collection load cycles. Severing the pubic symphysis ensured that applied loads were not shared or distributed throughout the pelvic ring but instead 
Table 2. $P$ values from range-of-motion analysis. ${ }^{a}$

\begin{tabular}{|c|c|c|c|}
\hline Type of Motion & 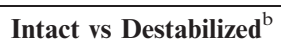 & Destabilized vs SI Instrumented & Intact vs SI Instrumented \\
\hline Flexion-extension & .003 & $<.001$ & .14 \\
\hline Lateral bending & $<.001$ & $<.001$ & .01 \\
\hline Axial rotation & $<.001$ & $<.001$ & .85 \\
\hline
\end{tabular}

Abbreviation: SI, sacroiliac.

${ }^{a}$ One-way repeated-measures analysis of variance and pairwise Holm-Š́dák tests. Bolded $P$ values indicate significance.

${ }^{\mathrm{b}} \mathrm{Cut}$ posterior ligament capsule and pubic symphysis.

were entirely supported by the individual left or right SI joint being tested. This step also permitted the pooling of left and right SI joint data for overall comparisons.

Application of the novel SI screw fixation device significantly reduced mean joint ROM as compared to the destabilized condition in all 3 anatomic planes of testing $(P<.001)$. When compared to the intact condition, joint ROM in the SI-instrumented condition was statistically significantly reduced $(P$ $=.01)$ in lateral bending, with mean values of $1.2 \pm$ $0.4^{\circ}$ and $0.6 \pm 0.3^{\circ}$, respectively. Although the mean observed SI-instrumented ROM of $1.8 \pm 1.0^{\circ}$ in flexion-extension was less than the intact ROM of $2.9 \pm 0.7^{\circ}$, the difference was not statistically significant $(P=.14)$. A relatively large $\mathrm{SD}$ and low sample size likely contributed to this outcome. In axial rotation, the mean $\mathrm{ROM}$ of $1.5 \pm 0.7^{\circ}$ observed in the SI-instrumented condition was statistically $(P=.85)$ and numerically equivalent to that observed for the intact joint condition $(1.5 \pm$ $0.3^{\circ}$ ).

Overall, SI joint stability was restored to a level equal to or less than that for the intact joint with application of the SI joint fixation device. For all directions tested, the mean SI-instrumented joint ROM was $\leq 1.8^{\circ}$. Previous biomechanical investigations of the stability of spinal fusion procedures using the gold standard of interbody implants supplemented with bilateral pedicle screw-and-rod instrumentation have typically reported an angular range of motion of $2^{\circ}$ for all directions tested under identical load conditions as those used in the current study. ${ }^{25,26}$ Although spinal fusion requirements may differ from those for the SI joint, the treated ROM values observed in the current study are comparable to levels of mobility considered favorable for spinal fusion.

The majority of previous biomechanical investigations of SI joint and pelvic stability have applied compressive loads, ${ }^{27-30}$ offset compressive loads, ${ }^{31}$ or combined compression with axial torsion. ${ }^{32,22}$
Those studies that included an applied torque have tended to report measures in terms of specimen stiffness and global specimen rotation ${ }^{32,33}$ as opposed to isolated SI joint rotation within anatomic planes. These differences in load application and outcome measures prevent a direct comparison of results from the current study. In one such recent study, Wu et $\mathrm{al}^{27}$ compared the in vitro biomechanical stability of Tile C pelvic fractures fixed with 2 iliosacral screws, tension band plating, and a novel minimally invasive adjustable plate under vertical compression loading. Although the loading mechanism was different than that applied in the current study, iliosacral screw fixation was reported to afford the strongest fixation of the 3 constructs tested.

With regard to previous biomechanical investigations of devices designed specifically for SI joint fusion, we are aware of only 2 other published reports. ${ }^{23,24}$ These studies were performed in our laboratory using the same in vitro testing protocol and measurement analysis as in the current study. In particular, the 2014 study by Lindsay et $\mathrm{al}^{23}$ applied an identical destabilization procedure as

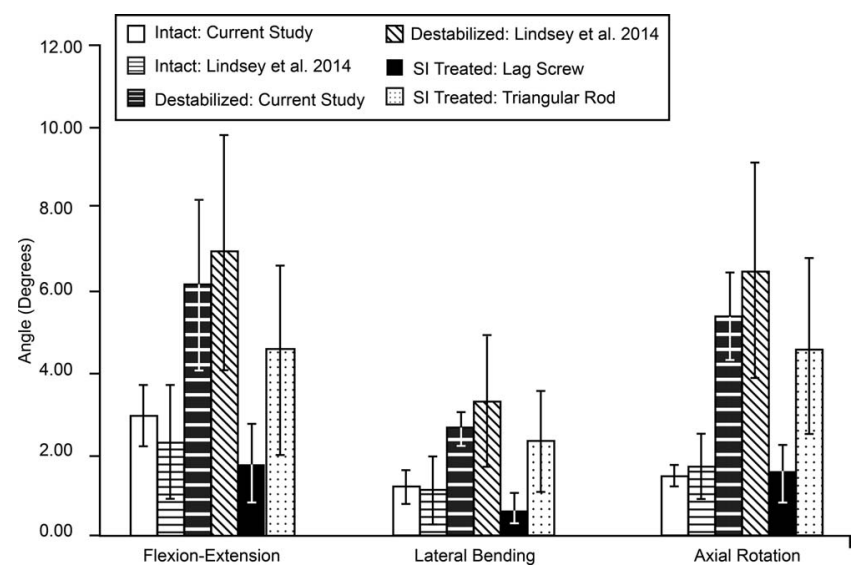

Figure 5. Mean angular range of motion observed at the sacroiliac (SI) joint from a previously published study by Lindsey et $\mathrm{a}^{23}$ compared to the same type of data from the current study. Error bars indicate standard deviations. Used with permission from Barrow Neurological Institute, Phoenix, Arizona. 
the one used in this study. Figure 5 presents a graphical comparison of results from Lindsey et al with those of the current study. Both the intact and the destabilized ROMs agreed well between the 2 studies for all directions of loading; this finding indicates the consistency and repeatability of the protocol application and the destabilization procedure. In the previous study, the destabilized condition produced mean increases in intact ROM of $206 \%$ in flexion-extension, $204 \%$ in lateral bending, and $287 \%$ in axial rotation as compared to increases for the destabilized versus the intact conditions in the current study of $111 \%$, $126 \%$, and $267 \%$, respectively, for the same directions of loading. In terms of SI-instrumented results, the condition investigated by Lindsey et $\mathrm{al}^{23}$ used a series of triangular implants (iFuse; SIBONE, Inc, San Jose, California) placed across the SI joint; these implants resulted in mean ROM reductions of $38 \%$ in flexion-extension, $31 \%$ in combined lateral bending, and $29 \%$ in axial rotation compared to the destabilized condition. The mean instrumented ROM reflected increases of $89 \%$ for flexion-extension, $110 \%$ for lateral bending, and $174 \%$ for axial rotation compared with the measured intact specimen ROMs. In comparison, the Integrity-SI construct in the current study, which used a large lag screw paired with a smaller antirotation screw placed across the SI joint, reflected mean ROM reductions of $72 \%$ in flexion-extension, $78 \%$ in combined lateral bending, and $72 \%$ in axial rotation compared to the destabilized condition. The instrumented condition also resulted in mean reductions of $40 \%$ and $49 \%$ of the intact specimen ROM in flexion-extension and combined lateral bending, respectively, and an increase of $4 \%$ over the intact specimen $\mathrm{ROM}$ in axial rotation.

The lag-screw configuration of the instrumented condition applied in the current study was designed to provide compression across the SI joint via screw thread purchase and a large variable-angle washer. No distraction of the SI joint occurs with application of this lag screw concept. Although compression was not measured directly, qualitatively the SI joint was observed to compress substantially, as noted by intraarticular contents extruding from the joint capsule. It is likely that joint compression as well as the rigidity of the instrumentation used were important factors that contributed to the overall instrumented stability observed under single-stance moment loads in the current study.

Compression and the resulting immediate stability of the SI joint leads to an optimal environment for true arthrodesis to occur. It is our belief that a principles-based system is optimal and necessary in order to provide a solid arthrodesis across the SI joint. As with other anatomical locations, solid arthrodesis can be challenging to achieve. Longterm results are predicated on achieving a solid fusion, and great care is needed to avoid a pseudarthosis or premature loosening or fracture of the implants, which may lead to poorer outcome and the need for revision surgery.

\section{Limitations}

Although the cadaveric specimens used in the current study were relatively young in age and of good quality, the sample size was small, and the use of matched pairs of SI joints may have limited specimen variability. The destabilized condition we used in this study was designed for comparative purposes and may not accurately reflect the nature of clinically presented instabilities. As with other cadaver studies, muscle forces were not simulated, and thus in vivo loads may vary. This study evaluated only the immediate stability of in vitro segmental fixation. Further clinical studies are needed to clarify performance of this novel fixation device and to contextualize results in the setting of clinical practice.

\section{CONCLUSIONS}

This study evaluated the biomechanical stability of initial SI joint fixation with a novel device using a previously published in vitro human cadaveric single-leg stance model. Intact SI joint ROMs were consistent with clinical observation, whereas destabilized ROMs were significantly increased in all 3 anatomic planes of testing. Instrumentation with the SI screw fixation device significantly reduced mean joint ROM compared to that for the destabilized condition, with similar ROM in flexion-extension and axial rotation, and it significantly reduced ROM in lateral bending compared to that for the intact joint. ROM values observed with the instrumented condition were comparable to levels of mobility considered favorable for spinal fusion. 


\section{ACKNOWLEDGMENTS}

Jakub Godzik, MD, Barrow Neurological Institute, performed destabilization procedures for the study.

\section{REFERENCES}

1. Bernard TN Jr, Kirkaldy-Willis WH. Recognizing specific characteristics of nonspecific low back pain. Clin Orthop Relat Res. 1987;(217):266-280.

2. Sembrano JN, Polly DW Jr. How often is low back pain not coming from the back? Spine (Phila Pa 1976). 2009;34(1):E27-E32.

3. Cohen SP, Chen Y, Neufeld NJ. Sacroiliac joint pain: a comprehensive review of epidemiology, diagnosis and treatment. Expert Rev Neurother. 2013;13(1):99-116.

4. Cher D, Polly D, Berven S. Sacroiliac joint pain: burden of disease. Med Devices (Auckl). 2014;7:73-81.

5. Manchikanti L, Abdi S, Atluri S, et al. An update of comprehensive evidence-based guidelines for interventional techniques in chronic spinal pain. Part II: guidance and recommendations. Pain Phys. 2013;16(suppl 2):S49-S283.

6. Ackerman SJ, Polly DW Jr, Knight T, Schneider K, Holt $\mathrm{T}$, Cummings J Jr. Comparison of the costs of nonoperative care to minimally invasive surgery for sacroiliac joint disruption and degenerative sacroiliitis in a United States commercial payer population: potential economic implications of a new minimally invasive technology. Clinicoecon Outcomes Res. 2014;6:283-296.

7. Buchowski JM, Kebaish KM, Sinkov V, Cohen DB, Sieber AN, Kostuik JP. Functional and radiographic outcome of sacroiliac arthrodesis for the disorders of the sacroiliac joint. Spine J. 2005;5(5):520-528; discussion 9.

8. Rudolf L. Sacroiliac joint arthrodesis-MIS technique with titanium implants: report of the first 50 patients and outcomes. Open Orthop J. 2012;6:495-502.

9. Whang P, Cher D, Polly D, et al. Sacroiliac joint fusion using triangular titanium implants vs non-surgical management: six-month outcomes from a prospective randomized controlled trial. Int J Spine Surg. 2015;9:6.

10. Schutz U, Grob D. Poor outcome following bilateral sacroiliac joint fusion for degenerative sacroiliac joint syndrome. Acta Orthop Belg. 2006;72(3):296-308.

11. Sturesson B, Uden A, Vleeming A. A radiostereometric analysis of the movements of the sacroiliac joints in the reciprocal straddle position. Spine (Phila Pa 1976). 2000;25(2):214-217.

12. Fortin JD, Kissling RO, O'Connor BL, Vilensky JA. Sacroiliac joint innervation and pain. Am J Orthop (Belle Mead NJ). 1999;28(12):687-690.

13. Vilensky JA, O’Connor BL, Fortin JD, et al. Histologic analysis of neural elements in the human sacroiliac joint. Spine (Phila Pa 1976). 2002;27(11):1202-1207.

14. Smith AG, Capobianco R, Cher D, et al. Open versus minimally invasive sacroiliac joint fusion: a multi-center comparison of perioperative measures and clinical outcomes. Ann Surg Innov Res. 2013;7(1):14.
15. Ledonio CG, Polly DW Jr, Swiontkowski MF. Minimally invasive versus open sacroiliac joint fusion: are they similarly safe and effective? Clin Orthop Relat Res. 2014;472(6):1831-1838.

16. Stark JG, Fuentes JA, Fuentes TI, Idemmilli C. The history of sacroiliac joint arthrodesis: a critical review and introduction of a new technique. Curr Orthop Pract. 2011;22(6):545-557.

17. Clissan DJ. The indications for inducing fusion at the ankle joint by operation; with description of two successful techniques. Aust N Z J Surg. 1949;19(1):64-71.

18. Kaiser SP, Gardner MJ, Liu J, Routt ML Jr, Morshed $\mathrm{S}$. Anatomic determinants of sacral dysmorphism and implications for safe iliosacral screw placement. $J$ Bone Joint Surg Am. 2014;96(14):e120.

19. Crawford NR, Brantley AG, Dickman CA, Koeneman EJ. An apparatus for applying pure nonconstraining moments to spine segments in vitro. Spine (Phila Pa 1976). 1995;20(19):2097-2100.

20. Panjabi MM. Biomechanical evaluation of spinal fixation devices: I. A conceptual framework. Spine (Phila $\mathrm{Pa}$ 1976). 1988;13(10):1129-1134.

21. Crawford NR, Dickman CA. Construction of local vertebral coordinate systems using a digitizing probe: technical note. Spine (Phila Pa 1976). 1997;22(5):559-563.

22. Crawford NR, Yamaguchi GT, Dickman CA. A new technique for determining 3-D joint angles: the tilt/twist method. Clin Biomech (Bristol, Avon). 1999;14(3):153-165.

23. Lindsey DP, Perez-Orribo L, Rodriguez-Martinez N, et al. Evaluation of a minimally invasive procedure for sacroiliac joint fusion - an in vitro biomechanical analysis of initial and cycled properties. Med Devices (Auckl). 2014;7:131-137.

24. Soriano-Baron H, Lindsey DP, Rodriguez-Martinez N, et al. The effect of implant placement on sacroiliac joint range of motion: posterior versus transarticular. Spine (Phila $\mathrm{Pa}$ 1976). 2015;40(9):E525-E530.

25. Nayak AN, Gutierrez S, Billys JB, Santoni BG, Castellvi AE. Biomechanics of lateral plate and pedicle screw constructs in lumbar spines instrumented at two levels with laterally placed interbody cages. Spine J. 2013;13(10):13311338.

26. Reis MT, Reyes PM, Bse, et al. Biomechanical evaluation of lateral lumbar interbody fusion with secondary augmentation. J Neurosurg Spine. 2016:1-7.

27. Wu T, Chen W, Li X, Zhang Q, Lv HZ, Zhang YZ. Biomechanical comparison of three types of internal fixation in a type $\mathrm{C}$ zone II pelvic fracture model. Int J Clin Exp Med. 2015;8(2):1853-1861.

28. Kemper AR, McNally C, Duma SM. Dynamic compressive response of the human pelvis axial loading of the sacroiliac joint. Biomed Sci Instrum. 2008;44:171-176.

29. Dienstknecht T, Berner A, Lenich A, et al. Biomechanical analysis of a transiliac internal fixator. Int Orthop. 2011;35(12):1863-1868.

30. Mears SC, Sutter EG, Wall SJ, Rose DM, Belkoff SM. Biomechanical comparison of three methods of sacral fracture fixation in osteoporotic bone. Spine (Phila $\mathrm{Pa}$ 1976). 2010;35(10):E392-E395.

31. Kim JH, Horton W, Hamasaki T, Freedman B, 
Whitesides TE Jr, Hutton WC. Spinal instrumentation for sacral-pelvic fixation: a biomechanical comparison between constructs ending with either S2 bicortical, bitriangulated screws or iliac screws. J Spinal Disord Tech. 2010;23(8):506512.

32. Yu B, Zheng Z, Zhuang X, et al. Biomechanical effects of transverse partial sacrectomy on the sacroiliac joints: an in vitro human cadaveric investigation of the borderline of sacroiliac joint instability. Spine (Phila Pa 1976). 2009;34(13):1370-1375.

33. Dawei T, Na L, Jun L, Wei J, Lin C. A novel fixation system for sacroiliac dislocation fracture: internal fixation system design and biomechanics analysis. Clin Biomech (Bristol, Avon). 2013;28(2):129-133.

Disclosures and COI: Nick Slater is an employee of CoorsTek Medical, LLC. Dr Sigurd Berven receives royalties from Stryker Corp and Medtronic, plc; is a consultant with Stryker Corp, Medtronic, plc, Globus Medical, Inc, RTI Surgical,
Inc, and CoorsTek Medical, LLC; and has stock ownership in Providence Medical Technology and Simpirica Spine, Inc. This study was funded by a research grant from CoorsTek Medical, LLC.

Corresponding Author: Brian P. Kelly, PhD, c/o Neuroscience Publications, Barrow Neurological Institute, St. Joseph's Hospital and Medical Center, 350 West Thomas Rd, Phoenix, AZ 85013. Phone: (602) 406-3593; Fax: (602) 406-4104; Email: Neuropub@dignityhealth.org.

Published 15 October 2018

This manuscript is generously published free of charge by ISASS, the International Society for the Advancement of Spine Surgery. Copyright (C 2018 ISASS. To see more or order reprints or permissions, see http://ijssurgery.com. 